\title{
Location of the upper border of the cavity excavated after the Deep Impact collision
}

\author{
Sergei I. Ipatov ${ }^{1,2} \dagger$, \\ ${ }^{1}$ Space Research Institute, Moscow, Russia \\ email: siipatov@hotmail.com \\ ${ }^{2}$ Catholic University of America, Washington DC, USA
}

\begin{abstract}
The distance between the pre-impact surface of Comet 9P/Tempel 1 and the upper border of the largest cavity with dust and gas under pressure excavated after the collision of the impact module of the Deep Impact spacecraft with the comet is estimated to be about $6 \mathrm{~m}$ if the diameter of the transient crater was about $200 \mathrm{~m}$. This result suggests that cavities containing dust and gas under pressure located a few meters below surfaces of comets can be frequent.
\end{abstract}

Keywords. comets: individual (Comet 9P/Tempel 1), comets: general

In 2005 the impact module of the Deep Impact (DI) spacecraft collided with Comet 9P/Tempel 1. Based on analysis of the images made during the first 13 minutes after this impact, Ipatov \& A'Hearn (2011) concluded that the triggered outburst of small particles and excavation of a large cavity with dust and gas under pressure began at $t_{e}=8 \mathrm{~s}$, where $t_{e}$ is the time after the DI collision. The largest cavity excavated after the collision could be relatively deep because a considerable excess ejection lasted during about $50 \mathrm{~s}$. Schultz et al. (2013) analyzed the images of Comet 9P/Tempel 1 made by the Stardust spacecraft and concluded that the diameter $d_{t c}$ of the DI transient crater was about $200 \mathrm{~m}$. Some authors support smaller values of $d_{t c}$ (up to $50 \mathrm{~m}$ ). Supposing the depth of a growing crater to be proportional to $t_{e}^{\gamma}$ (where $\gamma$ is about 0.25-0.4) during the intermediate stage of crater excavation and adding $1 \mathrm{~m}$ for the initial stage, Ipatov (2012b) estimated the depth of the DI crater at $t_{e}=8 \mathrm{~s}$ (at the time of the beginning of excavation of the main cavity) to be about $6 \mathrm{~m}$ for $d_{t c}=200 \mathrm{~m}$ and $4 \mathrm{~m}$ for $d_{t c}=100 \mathrm{~m}$. This depth is in accordance with the depth $(4-20 \mathrm{~m})$ of the initial sublimation front of the $\mathrm{CO}$ ice in the models of the explosion of Comet 17P/Holmes considered by Kossacki \& Szutowicz (2011). The porous structure of comets provides enough space for sublimation and testifies in favor of existence of cavities. Natural outbursts were observed for several comets (see references in Ipatov 2012a). Similarity of velocities of particles ejected at triggered and natural outbursts shows that these outbursts could be caused by similar internal processes in comets. Our studies testify in favor of that cavities with dust and gas under pressure located a few meters below surfaces of comets can be frequent.

\section{References}

Ipatov, S. I. 2012a, in: P. G. Melark (ed.), Comets: Characteristics, Composition and Orbits

(Nova Science Publishers), p. 101 (http://arxiv.org/abs/1103.0330)

Ipatov, S. I. 2012b, MNRAS. 423, 3474

Ipatov, S. I. \& A'Hearn, M. F. 2011, MNRAS. 414, 76

Kossacki, K. J. \& Szutowicz, S. 2011, Icarus. 212, 847

Schultz, P. H., Hermalyn, B., \& Veverka, J. 2013, Icarus. 222, 502

$\dagger$ Present address: Alsubai Est. for Scientific Studies, Doha, Qatar 